\section{Theoretical and Computational Fluid Dynamics}

Published Online May 2009, Pages 1-7

http://dx.doi.org/10.1007/s00162-009-0107-8

(c) 2009 Springer. Part of Springer Science+Business

Media

The original publication is available at http://www.springerlink.com
Archimer, archive institutionnelle de l'Ifremer http://www.ifremer.fr/docelec/

\title{
On instability of elliptical hetons
}

Mikhail Sokolovskiy ${ }^{1,{ }^{*}}$, Jacques Verron ${ }^{2},{\text { Xavier } \text { Carton }^{3} \text { and Vladimir Gryanik }}^{4}$

\author{
${ }^{1}$ Water Problems Institute of RAS, 3 Gubkina Str., 119333 Moscow, Russia \\ ${ }^{2}$ Laboratoire des Ecoulements Géophysiques et Industriels, UMR 5519, CNRS, BP53 X, 38041 Grenoble Cedex, \\ France \\ ${ }^{3}$ Laboratoire de Physique des Océans, UFR Sciences, UBO, 6 Av. Le Gorgeu, 29200 Brest, France \\ ${ }^{4}$ Alfred-Wegener-Institute for Polar and Marine Research, Bremerhaven, Germany \\ *: Corresponding author : Mikhail Sokolovskiy, email address : sokol@aqua.laser.ru
}

\begin{abstract}
:
Using the method of contour surgery, we examine the evolution of an initially vertically aligned elliptical heton. A classification of quasi-stable and unstable regimes for the case of two-layered vortex structure is suggested.
\end{abstract}

Keywords: Elliptical vortex - Vortex instability - Heton

PACS 47.32.C - 47.32.cd - 47.20.Cq

\section{Introduction}

The classic Kirchhoff [1] solution for the elliptical vortex patch with semi-axes $a$ and $b$ and vorticity $\omega$, which rotates as a solid body with a constant angular velocity $=\omega a b /(a+b) 2$ is known from 1876. Seventeen years later Love [2] showed that for $x=a / b>3$ this stationary solution is unstable. Recently, Mitchell and Rossi [3] have given a complete quantification of the regimes of linear and non-linear instability of the elliptical vortex with respect to the parameter $X$. Kirchhoff solution had multiple generalizations. In particular, - Chaplygin $[4,5]$, and later Kida [6], Dritschel [7], Dhanak and Marshall [8], Legras and Dritschel [9] and others have shown that the introduction of an ambient velocity field, linearly dependent on the coordinates (this is analogous to the affine transformation of coordinates, converting ellipses into ellipses), allows to construct a solution, defining the behaviour of a pulsating vortex, which rotates with variable velocity, and it is a time-dependent value of $\mathrm{X}$. 
- Polvani \& Flierl [10] have introduced a notion of a generalized Kirchhoff vortex for a system of $N$ embedded one into other elliptical patches. They have studied the stability of such a solution, and constructed diagrams of stable and unstable states in the space of external geometric parameters.

- Kozlov [11] has summarized the problem of an elliptical vortex for the case, when the effect of the "entrainment" due to the introduction of an "effective" bottom friction is taken into account. This mechanism initiates cyclonic-anticyclonic asymmetry (when $\omega$ changes its sign), which is observed in ocean and atmosphere conditions. In [11] there are given the conditions under which the model gives the particular cases of Kirchhoff, Chaplygin and Kida.

The above-named works investigate the dynamics of elliptical vortex patches in a homogeneous non-rotating fluid. These results may be automatically applied also to the case of rotating fluid under the condition of geostrophic balance (when the hydrodynamic pressure plays the role of the stream function).

In this paper, in the frames of quasi-geostrophic approximation [12] we examine the problem on the behaviour of two vortex patches in a stratified rotating fluid, which consists of two non-mixing layers of equal thickness. It is supposed also that in the initial moment elliptical vortices of the upper and bottom layers are identical kinematically, and are located strictly one over other, but they have opposite signs of $\omega$. So, such two-layer vortex represents a heton [13].

In [14], a stationary solution in the form of an axially symmetrical heton has been investigated to be stable with respect to small harmonic disturbances of the boundary at $\chi=1$. There is shown that at $\gamma=R / R_{d}>1.705$ ( $R$ - is a characteristic horizontal scale, $R_{d}$ - is the internal deformation Rossby radius [12]) may appear unstable modes. Thus, it is established that even the circular heton may be unstable with respect to the degree of the stratification. Let us note that similar results have been obtained independently in [15] and [16].

\section{Numerical modelling of evolution}

For elliptically shaped vortex patches $(\chi>1)$ it is possible to carry out only numerical study of stability. It should be noted, that the combination of the vertically aligned non-circular vortex patches of opposite cyclonic vorticity in the two-layer fluid, strictly speaking, doesn't realize the stationary state. In this case the quasi-elliptical pulsating vortex patches, rotating in different directions, are reference marks; each of them induces an external field for its partner, which changes periodically both in time and in space. Therefore, we will interpret the initial state represented as the combination of two vertically aligned ellipses as some instantaneous disturbed quasi-stationary state. Bellow, we give the results of numerical experiments for investigating the evolution of such vortices. We used the two-layer version of the Contour Dynamics Method (surgery) [14,17]. Before the discussion of the results we would like to note that $R_{d}=\sqrt{g^{\prime} h} / 2 f\left(g^{\prime}=g \Delta \rho / \rho_{0}, g\right.$ is the acceleration of gravity, $\Delta \rho$ difference of fluid densities in the bottom and upper layers, $h$ - characteristic vertical scale, $\rho_{0}$ - mean value of the density, $f$ - Coriolis parameter equal to a doubled rotation velocity of the whole plane). Thereby, the parameter $\gamma$ may be treated in two ways: as a stratification parameter $(\gamma \ll 1$ and $\gamma \gg 1$ being the limits of the strong and weak stratification, correspondingly), and as a geometrical characteristic of the typical size of the vortex with respect to the radius of deformation.

\subsection{General results}

In Figure 1, a diagram of the states of the initially elliptical heton in the space of variables $(\gamma, \chi)$ represents the result of numerous calculations. There are shown areas of existence both of stable quasiperiodic solutions, and of the solutions with different types of instability. Summary results are the following:

- In case of a strong stratification (or small relative sizes of vortex patches) the barotropic type of interaction is predominant, when the vortices from the different layers poorly interact one with other, and their evolution occurs essentially by the self-action.

- When the eccentricity of the initially elliptical vortices grows, in the frames of this class of motions, there takes place a consecutive transition from the quasi-stable states of the system to the unstable 


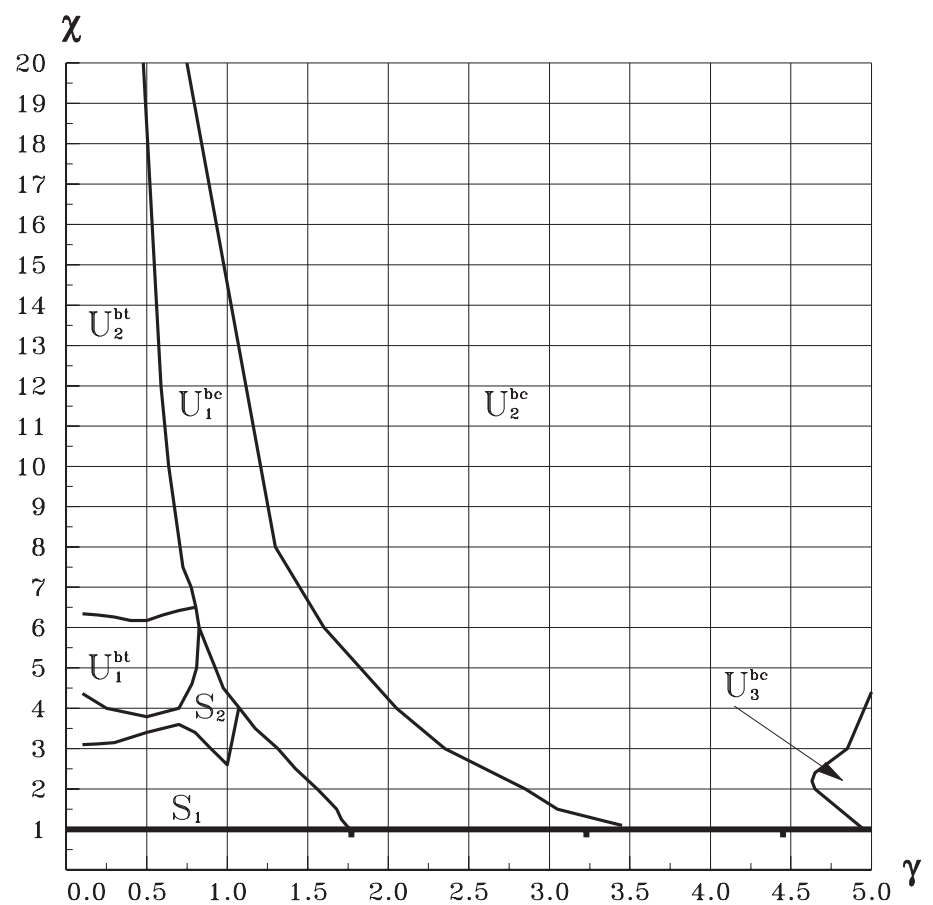

Fig. 1 Diagram of possible states of an elliptical heton with the vertical axis in a rectangular area $\gamma \in$ $(0,5], \chi \in[1,20]$. Symbols for sub-areas: $S_{1}$ - stable states; $S_{2}$ - quasi-stable states after a partial loss of the mass because of dropping of vortex threads; $U_{1}^{b t}$ - decay of the elliptical patches into non-symmetric parts because of the barotropic instability; $U_{2}^{b t}$ - decay of elliptical patches into symmetric parts because of the barotropic instability; $U_{1}^{b c}$ - decay of the elliptical patches into two hetons with tilted axes running away in the opposite directions because of the barotropic instability; $U_{2}^{b c}$ - cascade baroclinic instability with running away of a series of hetons along the main ellipse axis; $U_{3}^{b c}$ - cascade baroclinic instability with running away of hetons along both ellipse axes. Notches on the solid strait line $\chi=1$ divide the following areas for axially symmetrical heton: stability, and unstable mode with $m=2, m=3$ and $m=4$ (from left to right).

ones with non-symmetrical division of the vortex patches; and further $Ц$ to symmetrical division into two parts. In the same time the area of the quasi-barotropic motions shrinks.

- In case of a moderate stratification, a baroclinic type of interaction predominates with the realization of the vortex structure decay into two running in the opposite directions two-layer pairs with tilted axes. With the growth of ellipticity, the domain of existence with respect to the parameter $\gamma$ of this class of motions also significantly shrinks.

- In case of a strong stratification, the main mechanism of the progress of the process is the cascade instability, resulting in the further running away of more than two two-layer pairs.

\subsection{Analysis of realizations of different regimes}

When calculating, the potential vorticity of the upper-layer vortex patch was taken to be positive, and of the lower-layer one - negative. The absolute value of $\omega$ was defined in each of the experiments by such a way that a half of the rotational period of a fluid particle on the contour of a circular heton at the given value of $\gamma$ would correspond to a dimensionless unit of time .

Figure 2 shows the examples of calculations of the elliptical hetonsT evolution in the vicinity of the boundary of areas $S_{1}$ and $U_{1}^{b c}$ at the fixed values of the parameter $\chi=2$. In the first case a quasi-stable rotation of pulsating vortex patches in the upper and lower layers (barotropic type of interaction) occurs in the opposite directions. Vortex patches take a shape close to the elliptical one in the moment of the mutual overlapping (when the angular velocity of rotation is maximum) and the dumbbell shape when the conventional semi-axes have normal position (angular velocity is minimum in those moments). Note, that these effects - irregularities of rotation and deformation of contours - 


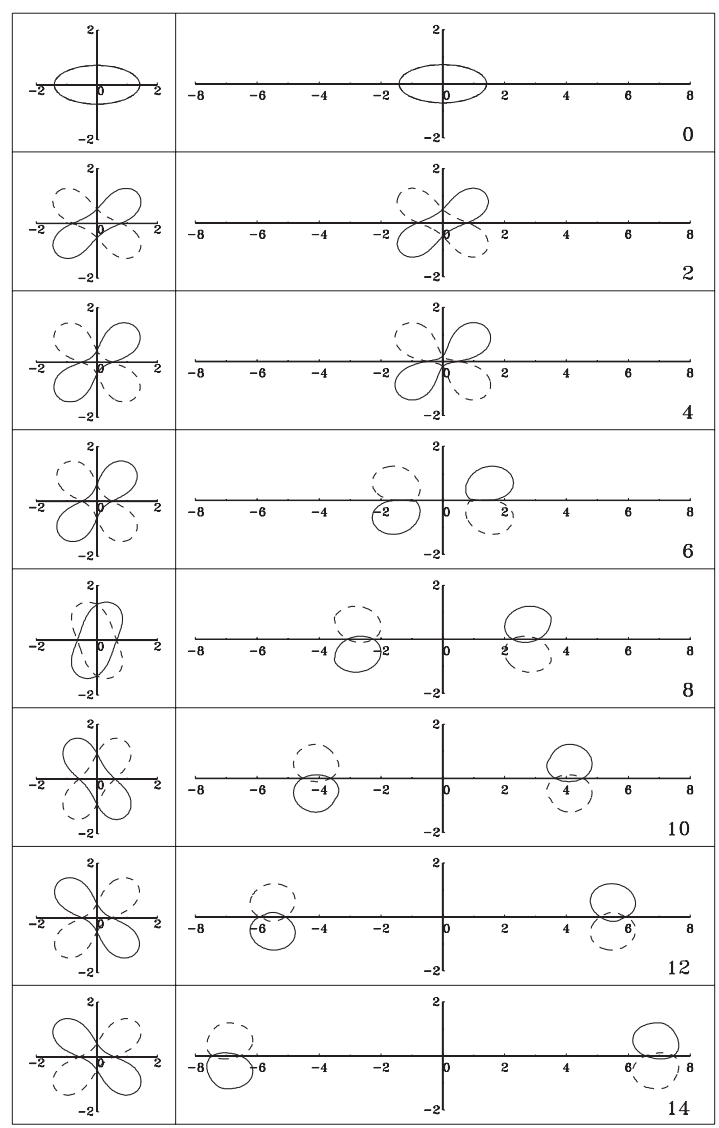

Fig. 2 Synchronous configurations of the quasi-stable (left, at $\gamma=1.56$ ) and unstable (right, at $\gamma=1.57$ ) elliptical hetons at $\chi=2$ at the indicated moments of dimensionless time. Solid and dashed lines depict contours of the vortex patches in the upper and bottom layer, correspondingly.

are expressed the stronger, the closer is the correspondent point of the plane $(\gamma, \chi)$ to the boundary of the stability and instability areas. In the second case, due to the mechanism of baroclinic instability, the initial structure decays into two running away two-layer pairs with tilted axes. Let us note the qualitative analogy of these variants with the case of two aligned point hetons [13], when the distance between them is either small, or large, correspondingly.

Figure 3 demonstrates the evolution on the non-linear stage of the barotropic instability for the non-symmetrical ( $U_{1}^{b t}$, left column) and symmetrical ( $U_{2}^{b t}$, central and right columns) types at a fixed value of $\gamma$ and different values of $\chi$ at the initial stage (before the separation of the vortex patches) and at the quasi-stationary stage of rotating movements of the vortices, that have being formed as a result of the decay of the initial vortex structure. The fact of the non-symmetrical decay of elliptical barotropic vortices at relatively small values of $\chi$ has been established for the first time in the numerical calculations by Kozlov and Makarov in [18].

In Figure 4 we illustrate the possibility of transition between the regimes of the cascade instability $U_{2}^{b c}$ and $U_{3}^{b c}$, when the parameter $\gamma$ increases. Note, that the initial evolution in the both variants runs identically, but in the moment $t=2$, the central part in the second case begins by now to stretch in the vertical direction, what ensure the further running away of small vortex patches in the opposite directions of the $y$ axis. This is the manifestation of the 4 -th mode formation.

Note that Figures 2 and 4 demonstrate a "robust" character of the vortex structures behaviour in dependence on the value of the parameter $\gamma$ : in the vicinity of the boundary between the different regimes an insignificant change of the stratification parameter causes the transition to the qualitatively another type of the vortex patchesT interaction. 


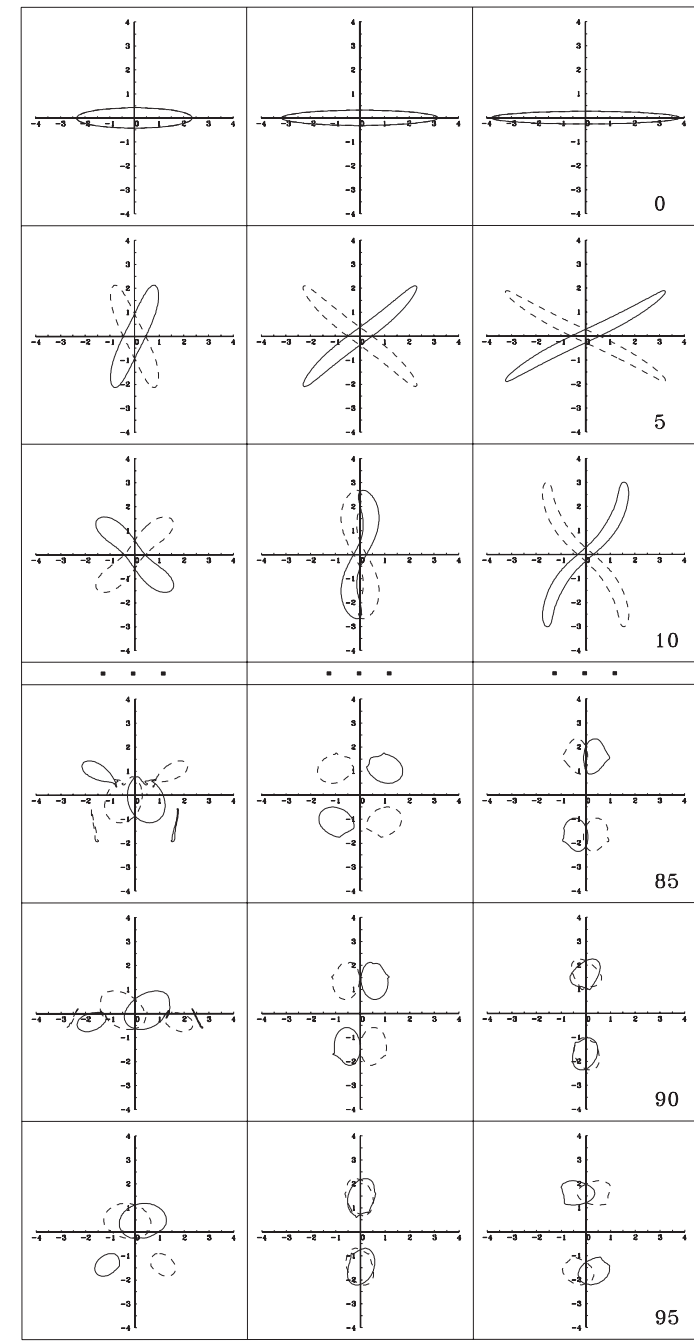

Fig. 3 Synchronous configurations of the non-symmetrical (left, at $\chi=5.5$ ) and symmetrical (central, at $\chi=$ 10 and right, at $\chi=14.5$ ) unstable elliptical hetons when $\gamma=0.25$ at the indicated moments of dimensionless time.

\section{Summary}

In the present work, the classification of stable and unstable regimes of elliptical hetons is constructed. We have shown that the possibility exists of two types of instability - barotropic and baroclinic ones, and that a weak stratification stimulates the conditions for cascading instability.

Acknowledgements We thank Hassan Aref and Dorte Glass for their care and hospitality. MS was supported by Russian Foundation for Basic Research (projects 07-05-92210, 07-05-00452 and 08-05-00061).

\section{References}

1. Kirchhoff, G.: Vorlesungen über matematische Physik: Mechanik. Taubner, Leipzig (1876).

2. Love, A.E.H.: On the stability of certain vortex motion. Proc. Lond. Math. Soc., 25, 18-42 (1893).

3. Mitchell, T.B., Rossi, L.F.: The evolution of Kirchhoff elliptical vortices. Phys. Fluids, 20, 054103 (2008).

4. Chaplygin, S.A.: On a pulsating cylindrical vortex. Trans. Phys. Sect. Imperial Moscow Soc. Frends of Nat. Sci., 10, 13-22 (1899).

5. Meleshko, V.V., van Heijst, G.J.F.: On Chaplygin's investigations of two-dimensional vortex structures in an inviscid fluid. J. Fluid Mech., 272, 157-182 (1994). 


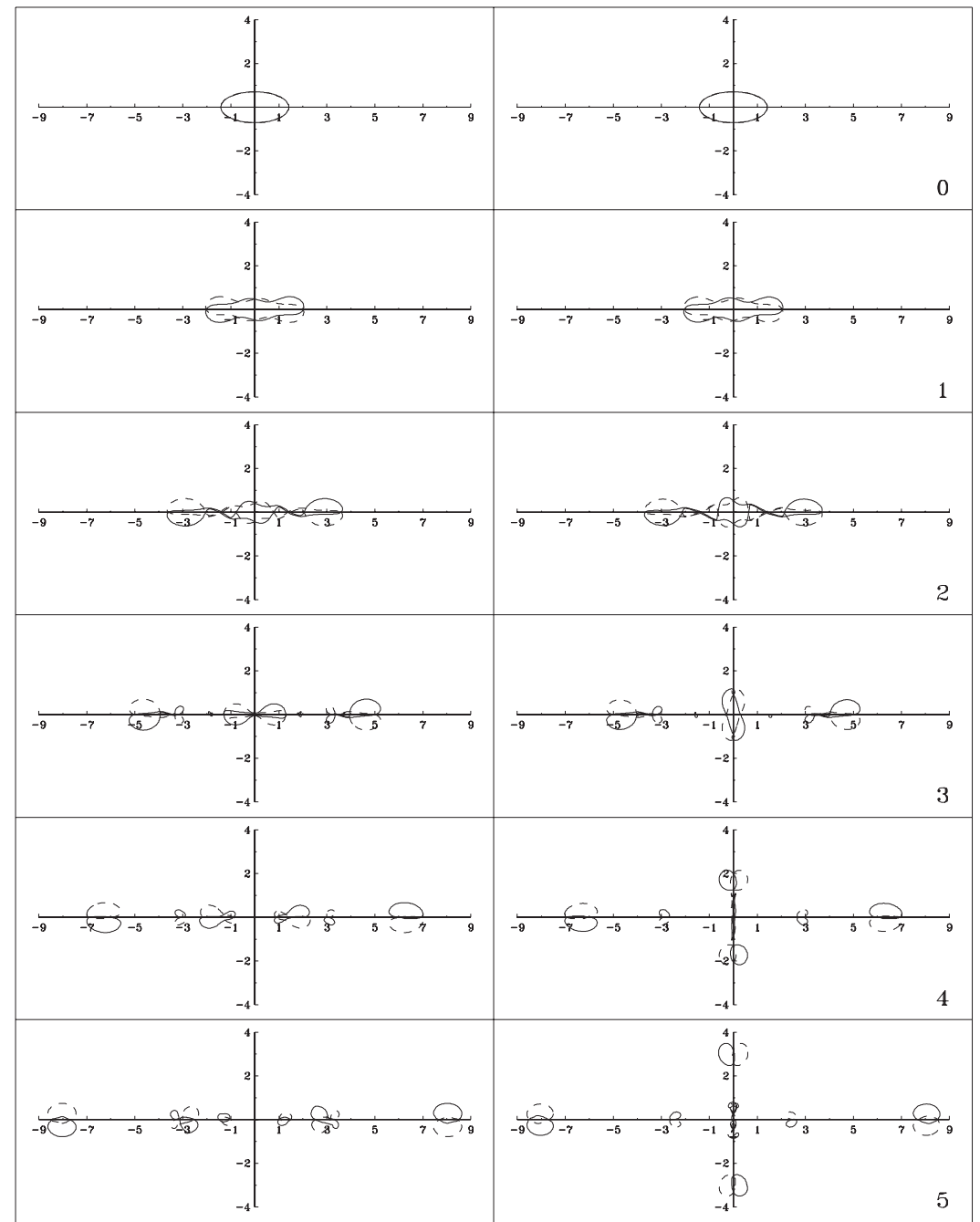

Fig. 4 Synchronous configurations of unstable elliptical hetons with vertical axes at $\chi=2$ and $\gamma=4.55$ on the left (type $U_{2}^{b c}$ ) and $\gamma=4.57$ on the right (type $U_{3}^{b c}$ ).

6. Kida, S.: Motion of an elliptical vortex in an uniform shear flow. J. Phys. Soc. Japan, 50, 3517-3520 (1981).

7. Dritschel, D.G.: The stability of elliptical vortices in an external straining flow. J. Fluid Mech., 210, 223-261 (1990).

8. Dhanak, M.R., Marshall, M.P.: Motion of an elliptical vortex under applied periodic strain. Phys. Fluids, A5, 1224-1230 (1993).

9. Legras, B., Dritschel, D.G.: The elliptical model of two-dimensional vortex dynamics. I. The basic state. Phys. Fluids, A3, 845-854 (1991).

10. Polvani, L.M., Flierl, G.R.: Generalized Kirchhoff vortices. Phys. Fluids, 29, 2376-2379 (1986).

11. Kozlov, V.F.: Model of two-dimensional vortex motion with an entrainment mechanism. Fluid Dynamics, 27, 793-798 (1991).

12. Pedlosky, J.: Geophysical fluid dynamics. 2nd ed. Springer (1987).

13. Hogg N.G., Stommel H.M.: The heton, an elementary interaction between discrete baroclinic geostrophic vortices, and its implications concerning eddy heat-flow. Proc. R. Soc. Lond., A 397, 1-20 (1985).

14. Kozlov, V.F., Makarov, V.G., Sokolovskiy, M.A.: A numerical model of baroclinic instability of axially symmetric vortices in a two-layer ocean. Izvestiya, Atmospheric and Oceanic Physics., 22, 868-874 (1986).

15. Pedlosky, J.: The instability of continuous heton clouds. J. Atmos. Sci. 42, 1477-1486 (1985).

16. Helfrich, K.R., Send, U.: Finite-amplitude evolution of two-layer geostrophic vortices. J. Fluid Mech., 197, 331-348 (1988).

17. Makarov, V.G.: Computational algorithm of the contour dynamics method with changeable topology of domains under study. Model. Mech. 5(22), 83-95 (1991). (In Russian)

18. Kozlov, V.F., Makarov, V.G.: Evolution modelling of unstable geostrophic eddies in a barotropic ocean. Oceanology, 24, 737-743 (1984). 